\title{
Impact of sirtuin-1 expression on H3K56 acetylation and oxidative stress: a double-blind randomized controlled trial with resveratrol supplementation
}

\author{
Simona Bo ${ }^{1}$. Gabriele Togliatto ${ }^{1} \cdot$ Roberto Gambino ${ }^{1} \cdot$ Valentina Ponzo $^{1} \cdot$ Giusy Lombardo $^{1} \cdot$ Rosalba Rosato $^{2}$. \\ Maurizio Cassader ${ }^{1} \cdot$ Maria Felice Brizzi ${ }^{1}$ (1)
}

Received: 13 November 2017 / Accepted: 29 December 2017 / Published online: 12 January 2018

(c) The Author(s) 2018. This article is an open access publication

\begin{abstract}
Aims Sirtuin-1 (SIRT-1) down-regulation in type 2 diabetes mellitus (T2DM) has been associated with epigenetic markers of oxidative stress. We herein aim to evaluate whether an increase in SIRT-1 expression affects histone 3 acetylation at the 56 lysine residue (H3K56ac) in T2DM patients randomly selected to receive either resveratrol $(40 \mathrm{mg}$ or $500 \mathrm{mg}$ ) or a placebo for 6 months. The primary outcome is changes in the H3K56ac level by variation in SIRT-1 expression and the secondary outcome is the evidence of association between SIRT-1 level, antioxidant markers (TAS), and metabolic variables.

Methods and results At baseline, peripheral blood mononuclear cell H3K56ac values among the SIRT-1 tertiles did not differ. At trial end, SIRT-1 levels were significantly higher in patients receiving $500 \mathrm{mg}$ resveratrol. At follow-up, patients were divided into tertiles of delta (trial end minus baseline) SIRT-1 value. Significant reductions in H3K56ac and body fat percentage were found in the highest tertile as were increased TAS levels. A multiple logistic regression model showed that the highest delta SIRT-1 tertile was inversely associated with variations in H3K56ac (OR $=0.66$; 95\% CI 0.44-0.99), TAS $(\mathrm{OR}=1.01 ; 95 \% \mathrm{CI} 1.00-1.02)$, and body fat percentage ( $\mathrm{OR}=0.75 ; 95 \%$ CI $0.58-0.96)$.

Conclusions We provide new knowledge on H3K56ac and SIRT-1 association in T2DM. These data suggest that boosting SIRT-1 expression/activation may impact redox homeostasis in these patients.

ClinicalTrials.gov Identifier NCT02244879.
\end{abstract}

Keywords Resveratrol $\cdot$ Epigenetics $\cdot$ PBMC $\cdot$ Sirtuin-1

\section{Introduction}

Chronic diseases, including obesity and diabetes mellitus (T2DM), correlate with redox homeostasis imbalance. Mitochondrial reactive oxygen species (ROS) generation is considered the major regulator of hyperglycemia-mediated epigenetic changes, including histone $(\mathrm{H})$ posttranslational modifications $[1,2]$. In particular, H3K terminal tail acetylation largely results in gene transcription after

Managed by Massimo Porta.

Maria Felice Brizzi

mariafelice.brizzi@unito.it

1 Department of Medical Sciences, University of Turin, Corso Dogliotti 14, 10126 Turin, Italy

2 Department of Psychology, University of Turin, Turin, Italy genotoxic damage [3, 4]. Lo et al. [5] have recently shown that the highest $\mathrm{H} 3$ acetylation level at the 56 lysine residue (H3K56ac) is associated with proteins and transcription factors involved in T2DM signaling pathways in half of the mature adipocyte genome. H3K56 is deacetylated by the class III histone deacetylase (HDAC) sirtuins [3, 4]. One sirtuin family member, SIRT-1, was found persistently downregulated in T2DM patients [6] and has been associated with mitochondrial ROS accumulation and increased p53 acetylation (p53ac) levels [7, 8]. Little data on the association between SIRT-1 and H3K56ac have been provided [3, 4, 9].

Resveratrol, 3,5,4'-trihydroxy-trans-stilbene, activates SIRT-1 and is a ROS scavenger [10]. Its benefits in human disease are highly controversial since not all the beneficial properties described in preclinical studies have been confirmed by clinical trials $[11,12]$, and concerns about its favorable effects were recently raised by systematic reviews and position statements [13-15]. We recently failed to detect 
any metabolic benefits of resveratrol in T2DM patients without vascular complications [16].

We therefore aimed to evaluate whether variations of SIRT-1 expression were associated with changes in $\mathrm{H} 3 \mathrm{~K} 56 \mathrm{ac}$ and p53ac levels, and/or with modifications of oxidative stress markers, inflammatory or metabolic variables in T2DM patients. Peripheral blood mononuclear cells (PBMCs) were used as surrogates to evaluate SIRT-1, H3K56ac, and p53ac content. Unexpectedly, not all patients receiving resveratrol showed increased SIRT-1 expression/ activation. Data were therefore analyzed by dividing participants according to increased SIRT-1 value, regardless of resveratrol treatment.

\section{Methods}

\section{Study design and intervention}

This was an observational study nested on a randomized controlled trial, which has previously been described [16, 17] and was registered at: www.clinicaltrials (Identifier: NCT02244879). Briefly, 192 T2DM patients were recruited from the Diabetic Clinic of the University of Turin from October 2013 through February 2016.

The inclusion criteria were: T2DM, age $\geq 40$ years, body mass index (BMI) $<35 \mathrm{~kg} / \mathrm{m}^{2}$, patients on diet and/or hypoglycemic agents other than insulin. Exclusion criteria were: treatment with any antioxidant substance, treatment with insulin, anticoagulants, steroids, or anti-inflammatory drugs different from aspirin, alcohol or substance abuse, uncompensated diabetes, liver or kidney diseases, presence of diabetes-related chronic complications, cardiovascular events or revascularization procedures in the previous 4-weeks, any severe chronic or life-threatening diseases, pregnancy, allergy to peanuts, grapes, wine, mulberries.

All patients recruited from March 2014 until February $2016(n=128)$ were enrolled for this study. All procedures were in agreement with the principles of the Helsinki Declaration; the study protocol was approved by the local ethics committee. All participants provided written informed consent.

Patients were randomized to one capsule/day of resveratrol $500 \mathrm{mg} /$ day (Resv500 arm), one capsule/day of resveratrol $40 \mathrm{mg}$ /day (Resv40 arm), and one capsule/day of placebo (totally inert microcellulose) (Placebo arm), respectively, for 6 months. Biotivia Bioceuticals (International SrL, Italy) prepared all the capsules, which were identical in size, shape, color, and taste. High-pressure liquid chromatography analyses revealed 99.7 and $97.9 \%$ trans-resveratrol purity in $500 \mathrm{mg}$ and $40 \mathrm{mg}$ capsules, respectively, and no resveratrol content in placebo capsules.
Patients and researchers who dispensed the capsules, collected data, and were involved in all measurements were blinded to the bottle content.

Participants were allocated as follows: 43,43 , and 42 in the Resv500, Resv40, and Placebo arms, respectively. All patients took medication in the morning and maintained their habitual lifestyle, the diet given by the Diabetic Clinic, their current hypoglycemic treatment and were instructed to abstain from nutritional supplements and significant amounts of resveratrol-rich foods and beverages $[12,16]$. Compliance with the study protocol was monitored with monthly phone calls and pill counting.

Patients were stratified by acetylsalicylic acid use and glycated hemoglobin (HbA1c) levels (cut-point 7\%), according to a computer-generated randomization sequence $[16,17]$.

\section{Outcomes}

Primary outcome Association between changes in SIRT-1 level and variation in $\mathrm{H} 3 \mathrm{~K} 56 \mathrm{ac}$ value.

Secondary outcomes Association between changes in SIRT-1 level and variation in p53ac, oxidative stress markers (total antioxidant status: TAS), anthropometric, metabolic, and inflammatory variables.

\section{Measurements}

Body weight and height were measured with light clothes and no shoes. Waist circumference was measured at the narrowest level by a plastic tape meter. Body composition was determined by dual X-ray densitometry (QDR-4500 Hologic Inc., Bedford, MA, USA), using whole-body absorptiometry software. Arterial blood pressure values were measured twice from the left arm, in a sitting position, after at least 10-min rest, with a mercury sphygmomanometer with appropriate cuff sizes (ERKA Perfect-Aneroid, Germany). The homeostasis model assessment-insulin resistance (HOMAIR) was calculated according to the published algorithm.

\section{Blood sample analysis}

Blood samples were freshly collected from each participant at baseline and at trial end. The laboratory measurements were blindly performed at the Laboratories of the Department of Medical Sciences, University of Turin.

The human PTX3 was measured by a ready-to-use solid-phase enzyme-linked immunosorbent assay based on the sandwich principle (Hycult biotech, Uden, The Netherlands). The intra-assay and inter-assay CVs were 3.6-3.8 and 4.1-4.9\%, respectively. TAS measurement was performed with a colorimetric assay (ImAnOx TAS Kit, 
Immundiagnostik AG Bensheim, Germany), with intra-assay and inter-assay CVs of 2.0-4.0 and 2.6-3.9\%, respectively.

Serum C-reactive protein (CRP) values were determined using a high-sensitivity latex agglutination assay on HITACHI 911 Analyzer (Sentinel Ch., Milan, Italy). The intra-assay and inter-assay CVs were $0.8-1.3$ and $1.0-1.5 \%$, respectively. Interleukin-6 (IL-6) circulating concentrations were measured by a quantitative sandwich enzyme immunoassay technique (R\&D System, Minneapolis, MN, USA) with an intra-assay CV of $6.9 \%$ and an inter-assay CV of $7.2 \%$.

Serum glucose was measured by the glucose oxidase method (Sentinel Ch., Milan) with an intra-assay CV of $1.1 \%$ and an inter-assay CV of $2.3 \%$. Triglycerides and cholesterol were assayed by enzymatic colorimetric assays (Sentinel Ch., Milan) with an intra-assay CV of $3.0 \%$ and an inter-assay $\mathrm{CV}$ of $3.5 \%$ for triglycerides and with an intra-assay CV of $2.2 \%$ and an inter-assay CV of $3.4 \%$ for cholesterol. HDL-cholesterol was determined by enzymatic colorimetric assay after precipitation of LDL and VLDL fractions using heparin-MnCl2 solution and centrifugation at $4{ }^{\circ} \mathrm{C}$, and it had an intra-assay $\mathrm{CV}$ of $2.5 \%$ and an inter-assay CV of $4.1 \%$. Free fatty acid (FFA) values were assayed by an enzymatic colorimetric method (RANDOX, UK).

Alanine aminotransferase (ALT) and y-glutamyltransferase (GGT) were measured by a kinetic determination (Sentinel Ch., Milan) according to the IFCC recommendations. Insulin was measured by a biotin labeled antibody based sandwich enzyme immunoassay (LDN, Germany). The kit had a sensitivity of less than $1.8 \mu \mathrm{U} /$ $\mathrm{ml}$ and a range of $0-100 \mu \mathrm{U} / \mathrm{ml}$. The intra-assay and interassay CVs were 1.8-2.6 and 2.9-6.0\%, respectively. Glycated hemoglobin (Hbalc) was determined with a latexbased method (Sentinel Ch., Milan). The intra-assay and inter-assay CVs were 1.1-1.5 and 1.1-1.6\%, respectively. Adiponectin was measured by sandwich enzyme-linked immunosorbent assays (BioVendor, Brno, Czech Republic). The kit has a sensitivity of $470 \mathrm{ng} / \mathrm{ml}$ and a range of 5000 to $150,000 \mathrm{ng} / \mathrm{ml}$. The intra- and inter-assay CVs were 4.1 and $6.9 \%$, respectively. Uric acid was assayed by uricase-based enzymatic colorimetric assays (Sentinel Ch., Milan) with an intra-assay CV of $2.1 \%$ and an inter-assay CV of $1.7 \%$.

\section{Peripheral blood mononuclear cell (PBMC) preparation}

Blood sample (14 mL) was freshly collected from each patient participating in the randomized controlled trial (RCT) at baseline and at the end of treatment. PBMCs were isolated by Ficoll-Paque (Histopaque 1077; SigmaAldrich) density gradient centrifugation (400 g for $30 \mathrm{~min}$ at RT), washed twice with saline phosphate buffer (PBS) ( $300 \mathrm{~g}$ for $10 \mathrm{~min}$ at $4{ }^{\circ} \mathrm{C}$ ), and lysed using a lysis buffer for nuclear protein extraction.

\section{Western blot analysis}

PBMCs were lysed as previously described [18]. Proteins $(50 \mu \mathrm{g})$ were subjected to SDS-PAGE, transferred into nitrocellulose membrane, blotted with the indicated antibodies, and processed. Densitometric analysis was used to calculate the differences in the fold induction of protein levels normalized to $\beta$-actin, H3, and p53 content. Twenty samples, randomly selected, were double analyzed, and similar results were obtained. Values are reported as relative amounts (ra). All experiments were blindly performed.

\section{Statistics}

Within-group differences among the three arms were evaluated using the Wilcoxon matched-pairs test, while betweengroup differences were computed using the Kruskal-Wallis test.

Participants were divided according to tertiles of the baseline SIRT-1 values; the highest tertile (SIRT-1 $\geq 1.16 \mathrm{ra}$ ) was compared with the other two. The differences between end-of-the-trial values and each variable's baseline value (delta) were calculated. Delta SIRT-1 tertiles were then computed; analyzed variable delta values were compared with the highest tertile of delta SIRT-1 ( $\geq 0.66 \mathrm{ra})$ and the other two.

Either the Student's $t$ test or the Mann-Whitney $U$ test (non-normally distributed variables) was used to investigate differences in the variables between SIRT-1 value tertiles.

Associations between the highest tertile of delta SIRT-1 values and the median values of delta H3K56ac, delta TAS, and delta percent body fat were evaluated using a multiple logistic regression model, after adjustments for age, gender, and resveratrol treatment.

The power of the study, calculated by using the Wilcoxon test for delta H3K56ac mean values, was 0.82 with $\alpha$-value $=0.05$.

\section{Results}

\section{Baseline expression of SIRT-1 and its targets, H3K56ac and p53ac}

Mean \pm SD and median (interquartile range) baseline values of SIRT-1 in T2DM patients were $1.04 \pm 0.71$ and 0.86 (0.93) ra. Table 1 shows patient characteristics, by SIRT-1 
Table 1 Baseline characteristics of T2DM patients by SIRT-1 tertiles

\begin{tabular}{|c|c|c|c|}
\hline Baseline variables & Lower tertiles $(<1.16 \mathrm{ra})$ & Highest tertile $(\geq 1.16 \mathrm{ra})$ & $P^{*}$ \\
\hline Number & 86 & 42 & \\
\hline Males (\%) & 67.4 & 54.8 & 0.16 \\
\hline Age (years) & $65.6 \pm 7.3$ & $64.0 \pm 9.4$ & 0.31 \\
\hline Weight $(\mathrm{kg})$ & $80.3 \pm 12.5$ & $83.8 \pm 15.8$ & 0.18 \\
\hline $\mathrm{BMI}\left(\mathrm{kg} / \mathrm{m}^{2}\right)$ & $29.1 \pm 3.7$ & $30.2 \pm 3.6$ & 0.13 \\
\hline Waist circumference $(\mathrm{cm})$ & $102.7 \pm 9.7$ & $105.5 \pm 11.3$ & 0.15 \\
\hline Percent body fat & $33.5 \pm 6.7$ & $35.6 \pm 7.3$ & 0.10 \\
\hline Systolic blood pressure $(\mathrm{mmHg})$ & $132.1 \pm 10.1$ & $132.1 \pm 8.4$ & 0.98 \\
\hline Diastolic blood pressure $(\mathrm{mmHg})$ & $80.7 \pm 8.2$ & $80.4 \pm 7.6$ & 0.87 \\
\hline Fasting glucose (mg/dl) & $138.8 \pm 33.3$ & $145.0 \pm 43.5$ & 0.37 \\
\hline \multirow[t]{2}{*}{ Glycated hemoglobin $(\%)(\mathrm{mmol} / \mathrm{mol})$} & $6.75 \pm 0.86$ & $7.16 \pm 1.34$ & 0.038 \\
\hline & $50.3 \pm 9.4$ & $54.7 \pm 14.6$ & \\
\hline Fasting C-peptide (nmol/l) & $0.95 \pm 0.44$ & $1.05 \pm 0.48$ & 0.21 \\
\hline Fasting insulin $(\mu \mathrm{U} / \mathrm{ml})^{* *}$ & $16.2(11.0)$ & $18.2(10.6)$ & 0.34 \\
\hline HOMA-IR $(\mathrm{mmol} / \mathrm{L} \times \mu \mathrm{U} / \mathrm{ml}) * *$ & $5.29(4.03)$ & $5.41(4.02)$ & 0.26 \\
\hline Total cholesterol (mg/dl) & $176.6 \pm 37.9$ & $184.6 \pm 40.9$ & 0.28 \\
\hline Triglycerides $(\mathrm{mg} / \mathrm{dl})^{* *}$ & $106.5(86.0)$ & $111.0(77.0)$ & 0.52 \\
\hline HDL-cholesterol (mg/dl) & $46.7 \pm 12.9$ & $44.6 \pm 10.2$ & 0.38 \\
\hline LDL-cholesterol (mg/dl) & $103.1 \pm 34.3$ & $112.9 \pm 34.0$ & 0.13 \\
\hline Free fatty acids (mmol/l) & $0.66 \pm 0.19$ & $0.69 \pm 0.19$ & 0.40 \\
\hline Pentraxin-3 (ng/ml)** & $0.81 \pm 0.58$ & $0.70 \pm 0.39$ & 0.29 \\
\hline Interleukin-6 $(\mathrm{pg} / \mathrm{ml})^{* *}$ & $2.83(2.38)$ & $2.41(1.43)$ & 0.13 \\
\hline C-reactive protein $(\mathrm{mg} / \mathrm{dl})^{* *}$ & $1.52(2.47)$ & $1.56(3.67)$ & 0.66 \\
\hline Adiponectin $(\mathrm{ng} / \mathrm{ml})^{* *}$ & $7545.1(4746.5)$ & $7188.9(5974.1)$ & 0.72 \\
\hline Total antioxidant status $(\mu \mathrm{mol} / \mathrm{L})$ & $294.2 \pm 41.0$ & $291.3 \pm 41.4$ & 0.71 \\
\hline Uric acid (mg/dl) & $5.7 \pm 1.4$ & $5.4 \pm 1.2$ & 0.23 \\
\hline Alanine aminotransferase (U/l) & $19.5 \pm 9.6$ & $18.7 \pm 8.9$ & 0.65 \\
\hline y-glutamyl-transferase $(\mathrm{U} / \mathrm{l}) * *$ & $23.0(19.0)$ & $25.0(18.0)$ & 0.83 \\
\hline $\mathrm{H} 3 \mathrm{~K} 56 \mathrm{ac}(\mathrm{ra}) * *$ & $0.88(0.85)$ & $0.96(1.12)$ & 0.39 \\
\hline p53ac (ra) & $0.78 \pm 0.53$ & $0.90 \pm 0.64$ & 0.27 \\
\hline
\end{tabular}

$B M I$ body mass index, HOMA-IR homeostasis model assessment-insulin resistance, $r a$ relative amount *P obtained by Student's $t$ test or Chi-square, as appropriate

**Median (interquartile range); $P$ obtained by Mann-Whitney $U$ test tertiles at baseline (the highest tertile was compared to the lowest two tertiles). We found that patients in the highest tertile showed higher HbA1c values. No differences in $\mathrm{H} 3 \mathrm{~K} 56 \mathrm{ac}$ and p53ac values were detected in the two groups.

\section{Increased SIRT-1 expression correlates with a reduction in $\mathrm{H} 3 \mathrm{~K} 56 \mathrm{ac}$, but not p53ac}

The following dropouts occurred at follow-up: two (Placebo arm), two (Resv40 arm), one (Resv500 arm). At trial end, the mean $\pm \mathrm{SD}$ and median (interquartile range) values of SIRT-1 were $1.37 \pm 1.65$ and 0.97 (1.23) ra. The mean delta SIRT-1 value was $0.36 \mathrm{ra}$. The values of SIRT-1, by trial arm, are reported in Table 2. Within-group SIRT-1 values significantly increased at trial end in the Resv500 group ( $p<0.001$ by Wilcoxon test), and the delta values differed among the groups. Median H3K56ac and p53ac values did not differ among arms of treatment. The median delta values for each analyzed variable, by delta SIRT-1 tertiles, are shown in Table 3 (left side). A significantly higher proportion of Resv500 arm patients showed higher increases in SIRT-1 value. Patients in the highest delta SIRT-1 tertile displayed a significant decrease in H3K56ac. No significant differences in p53ac expression levels were detected. SIRT-1, H3K56ac, and p53ac expression levels for six patients in each arm are reported in Figs. 1 and 2.

The deltas of the different variables, by SIRT-1 variation, were calculated after excluding patients from the Placebo arm (Table 2, right side). Patients in the highest SIRT-1 value tertile still displayed a significant reduction in H3K56ac levels. 
Table 2 Median values of SIRT-1, H3K56ac, and P53ac, expressed as relative amounts, after treatment with resveratrol or placebo

\begin{tabular}{lrlll}
\hline & Placebo & Resv40 & Resv500 & $P$ \\
\hline SIRT-1 & & & & \\
Baseline & $0.93(1.00)$ & $0.81(1.01)$ & $0.79(0.79)$ & 0.74 \\
Trial end & $0.79(0.82)$ & $0.90(1.18)$ & $1.16(1.64)$ & 0.14 \\
Delta & $-0.16(1.33)$ & $0.01(1.36)$ & $0.45(1.21)$ & 0.046 \\
H3K56ac & & & & \\
Baseline & $0.87(1.06)$ & $0.85(1.02)$ & $1.04(0.96)$ & 0.56 \\
Trial end & $1.09(0.69)$ & $1.16(0.86)$ & $1.27(0.93)$ & 0.90 \\
Delta & $0.34(1.18)$ & $0.12(1.04)$ & $0.03(1.26)$ & 0.70 \\
P53ac & & & & \\
Baseline & $0.89(0.76)$ & $0.79(0.85)$ & $0.51(1.01)$ & 0.06 \\
Trial end & $0.81(0.69)$ & $1.06(0.99)$ & $0.93(0.78)$ & 0.25 \\
Delta & $0.06(0.60)$ & $0.22(0.92)$ & $0.19(0.88)$ & 0.94 \\
\hline
\end{tabular}

Delta end of trial minus baseline value; median (interquartile range)

$P$ values obtained by Kruskal-Wallis test

In a multiple logistic regression model, the highest delta SIRT-1 tertile was inversely associated with H3K56ac variation and directly related to resveratrol supplementation in all patients (Table 4). These associations were no longer statistically significant after excluding patients from the Placebo arm.

\section{Increased SIRT-1 expression was directly associated with total antioxidant status}

Highest delta SIRT-1 tertile patients showed significant increments in TAS values (Table 3), and that were still evident after excluding Placebo arm individuals. The direct association between median delta SIRT-1 values and delta TAS was confirmed by multiple regression analyses (Table 4).

\section{Comparison between SIRT-1 expression and metabolic and inflammatory variables}

Individuals in the highest SIRT-1 tertile showed a significant percentage body fat decrease (Table 3). This significant inverse relationship was confirmed using a multiple regression model (Table 4), but not after excluding the Placebo group.

No other metabolic variable was associated with changes in SIRT-1 expression. Similarly, no association between SIRT-1 change and inflammatory variables was found.

\section{Discussion}

We herein report that, at 6-month follow-up, increased SIRT-1 expression was associated with significant H3K56ac content reduction and increased serum antioxidant activity in T2DM patients.

SIRT-1 is a nicotinamide adenine dinucleotide (NAD)dependent deacetylase, involved in the deacetylation of histones and nonhistone proteins, including transcription factors, proteins and enzymes implicated in cell differentiation, cell survival, and metabolism [19, 20]. p53 was the first SIRT-1 target to be identified [21]. However, SIRT-1 also deacetylates $\mathrm{H} 3 \mathrm{~K} 56$, a crucial regulator of chromatin assembly/disassembly, which mainly marks transcriptionally active genes after genotoxic cues [3, 4]. More recently, genome-wide analysis demonstrated that highest levels of H3K56 acetylation are associated with diabetesrelated genes [5]. Indeed, changes in histone acetylation have a central role in sustaining the long-lasting effects of hyperglycemia mechanistically defined as "metabolic memory" [22]. As a matter of fact, SIRT1 also controls mitochondrial function and biogenesis, and free radical formation, key cues associated with epigenome reprogramming in diabetes [23]. Resveratrol is one of the most active polyphenols to stimulate SIRT-1 activity [24], which is known to be reduced in T2DM patients [6]. Therefore, we aimed to investigate whether rescue of SIRT-1 expression/activation could influence diabetes-related oxidative stress via H3K56 deacetylation. Unexpectedly, we found that not all T2DM patients receiving resveratrol displayed increased SIRT-1 expression/activation. Nevertheless, the increase in SIRT-1 expression was associated with changes in $\mathrm{H} 3 \mathrm{~K} 56 \mathrm{ac}$.

PBMCs were used as surrogates to evaluate SIRT-1 expression, while p53 and H3K56 acetylation were used as markers of its activation. A significant and dose-dependent increase in SIRT-1 expression was found after resveratrol supplementation. However, not all resveratrol arm patients showed an increase in SIRT-1 value at trial end. Changes in lifestyle and/or dietary intake, such as resveratrol-rich foods and beverages during the trial, may have caused unpredictable environmental conditions which may have influenced SIRT-1 expression. Moreover, genetically determined variants of the individual response to resveratrol treatment may also be postulated. Finally, the possibility of SIRT-1 being a direct target of resveratrol has been questioned [25], while both SIRT-1-independent effects [26] and decreased SIRT-1 levels [27] have been reported after resveratrol treatment.

Particular attention has recently been dedicated to the identification of oxidative stress-induced epigenetic T2DM markers [8]. Indeed, increased HDAC3 activity in PBMCs from T2DM patients has been directly correlated with 
Table 3 Median value of deltas (end-of-trial value minus baseline value) for each of the analyzed variables by tertiles of delta SIRT-1 in all participants (left side) and after excluding individuals from the Placebo arm (right side)

\begin{tabular}{|c|c|c|c|c|c|c|}
\hline & Lower tertiles $(<0.66 \mathrm{ra})$ & Highest tertile $(\geq 0.66 \mathrm{ra})$ & $P^{*}$ & Lower tertiles & Highest tertile & $P^{*}$ \\
\hline Number & 83 & 40 & & 50 & 33 & \\
\hline SIRT-1 (ra) & $-0.23(0.73)$ & $1.29(0.91)$ & $<0.001$ & $-0.14(0.53)$ & $1.28(1.04)$ & $<0.001$ \\
\hline Placebo arm (\%) & 39.8 & 17.5 & & - & - & \\
\hline Resv40 arm (\%) & 33.7 & 32.5 & & 56.0 & 39.4 & \\
\hline Resv500 arm (\%) & 26.5 & 50.0 & 0.014 & 44.0 & 60.6 & 0.14 \\
\hline Males (\%) & 61.5 & 70.0 & 0.35 & 60.0 & 63.6 & 0.74 \\
\hline Weight (kg) & $-0.50(2.50)$ & $-0.10(3.75)$ & 0.82 & $-0.25(3.00)$ & $-0.20(4.00)$ & 0.77 \\
\hline $\operatorname{BMI}\left(\mathrm{kg} / \mathrm{m}^{2}\right)$ & $0.00(0.90)$ & $-0.057(1.35)$ & 0.82 & $0.00(1.28)$ & $-0.09(1.39)$ & 0.43 \\
\hline Waist circumference $(\mathrm{cm})$ & $1.75(6.75)$ & $0.00(5.00)$ & 0.55 & $0.00(4.50)$ & $1.50(6.50)$ & 0.52 \\
\hline Percent body fat & $0.20(1.80)$ & $-0.60(3.00)$ & 0.018 & $0.00(1.80)$ & $-0.45(3.00)$ & 0.13 \\
\hline Systolic blood pressure $(\mathrm{mmHg})$ & $0.00(12.0)$ & $0.00(12.5)$ & 0.71 & $0.00(10.0)$ & $0.00(10.0)$ & 0.41 \\
\hline Diastolic blood pressure (mmHg) & $0.00(10.0)$ & $0.00(15.0)$ & 0.60 & $0.00(12.0)$ & $0.00(10.0)$ & 0.17 \\
\hline Fasting glucose (mg/dl) & $2.00(27.0)$ & $-2.00(24.5)$ & 0.32 & $2.00(20.0)$ & $1.00(21.0)$ & 0.28 \\
\hline \multirow{2}{*}{$\begin{array}{l}\text { Glycated hemoglobin }(\%)(\mathrm{mmol} / \\
\text { mol) }\end{array}$} & $0.30(1.40)$ & $0.25(1.15)$ & 0.20 & $0.35(1.30)$ & $0.20(1.10)$ & 0.22 \\
\hline & $3.28(15.3)$ & $2.73(12.6)$ & & $3.83(14.2)$ & $2.19(12.0)$ & \\
\hline Fasting C-peptide (nmol/l) & $-0.040(0.43)$ & $-0.06(0.47)$ & 0.45 & $-0.02(0.47)$ & $-0.08(0.47)$ & 0.21 \\
\hline Fasting insulin $(\mu \mathrm{U} / \mathrm{ml})$ & $2.89(9.76)$ & $0.32(9.29)$ & 0.26 & $2.89(12.8)$ & $1.81(9.26)$ & 0.28 \\
\hline HOMA-IR $(\mathrm{mmol} / \mathrm{L} \times \mu \mathrm{U} / \mathrm{ml})$ & $1.04(3.75)$ & $0.17(3.39)$ & 0.22 & $1.13(5.03)$ & $0.28(2.94)$ & 0.32 \\
\hline Total cholesterol (mg/dl) & $-7.50(43.0)$ & $-1.00(48.0)$ & 0.31 & $-4.00(38.0)$ & $-3.00(50.0)$ & 0.76 \\
\hline Triglycerides (mg/dl) & $13.00(55.0)$ & $12.50(45.5)$ & 0.66 & $16.0(71.0)$ & $14.0(46.0)$ & 0.40 \\
\hline HDL-cholesterol (mg/dl) & $2.00(12.0)$ & $2.50(10.0)$ & 0.37 & $2.00(12.0)$ & $3.00(11.0)$ & 0.16 \\
\hline LDL-cholesterol (mg/dl) & $-6.50(34.0)$ & $-4.50(41.5)$ & 0.48 & $-3.50(32.0)$ & $-3.00(43.0)$ & 0.95 \\
\hline FFA (mmol/l) & $0.01(0.04)$ & $0.01(0.04)$ & 0.93 & $0.01(0.06)$ & $0.01(0.04)$ & 0.79 \\
\hline Pentraxin-3 (ng/ml) & $0.04(0.60)$ & $0.08(0.62)$ & 0.82 & $0.13(0.71)$ & $0.00(0.60)$ & 0.36 \\
\hline Interleukin-6 (pg/ml) & $0.19(1.64)$ & $-0.27(1.60)$ & 0.16 & $0.32(1.84)$ & $-0.23(1.70)$ & 0.33 \\
\hline C-reactive protein $(\mathrm{mg} / \mathrm{dl})$ & $-0.02(2.51)$ & $0.00(2.22)$ & 0.38 & $0.00(2.63)$ & $0.01(2.22)$ & 0.54 \\
\hline Adiponectin (ng/ml) & $137.4(3583.8)$ & $653.0(3555.4)$ & 0.71 & $-449.6(3763.8)$ & $463.3(3217.0)$ & 0.43 \\
\hline Total antioxidant status $(\mu \mathrm{mol} / \mathrm{L})$ & $-2.00(62.0)$ & $13.5(72.0)$ & 0.033 & $4.00(70.0)$ & $25.0(66.0)$ & 0.047 \\
\hline Uric acid (mg/dl) & $0.00(1.40)$ & $0.30(1.25)$ & 0.32 & $0.05(1.50)$ & $0.30(1.20)$ & 0.44 \\
\hline $\operatorname{ALT}(\mathrm{U} / \mathrm{l})$ & $2.00(11.0)$ & $-1.00(12.5)$ & 0.12 & $1.00(13.0)$ & $-1.00(14.0)$ & 0.21 \\
\hline GGT (U/l) & $1.00(7.00)$ & $0.00(8.00)$ & 0.68 & $2.00(9.00)$ & $0.00(9.00)$ & 0.29 \\
\hline H3K56ac (ra) & $0.34(0.95)$ & $-0.20(1.40)$ & 0.025 & $0.25(1.04)$ & $-0.22(1.37)$ & 0.028 \\
\hline P53ac (ra) & $0.10(0.80)$ & $0.32(1.02)$ & 0.24 & $0.18(0.74)$ & $0.40(1.30)$ & 0.50 \\
\hline
\end{tabular}

Median (interquartile range) $* P$ obtained by Mann-Whitney $U$ test or Chi-square, as appropriate

$B M I$ body mass index, HOMA-IR homeostasis model assessment-insulin resistance, FFA free fatty acids, ALT alanine aminotransferase, GGT y-glutamyl-transferase, $r a$ relative amount

inflammation and insulin resistance, and negatively with SIRT-1 level [28]. Accordingly, HDAC activity suppression in embryos subjected to hyperglycemia-induced oxidative stress resulted in increased H3K56ac level [29]. H3K56ac has been linked to the activation of genes related to the unbalance of redox signals $[3,5,9]$. The inverse relationship between SIRT-1 level and H3K56ac is consistent with the original observation that antioxidant treatments reduced H3K56ac [3]. The significant correlation between increased serum antioxidant activity and SIRT-1/H3K56ac expression may be a proof of concept.
T2DM is characterized by low-grade chronic inflammation, which has been linked to low sirtuin levels [30]. A close connection between SIRT-1 and p53ac has been reported, primarily in diabetes-associated vascular dysfunction/inflammation [8, 30]. Long-lasting diabetes (median value: $8-y$ ) is a relevant feature in our patients. An impaired adaptive SIRT-1-mediated counter-acting response to environmental cues may therefore be a possible explanation for the failure to find correlation between SIRT-1, p53ac, and inflammatory parameters. Alternatively, the low sample size may have masked small, but 
Fig. 1 SIRT-1, H3K56ac, and p53ac protein expression in PBMCs recovered from T2DM patients submitted to resveratrol supplementation or placebo. a Cell extracts from T2DM patients' PBMCs (subject 1-6) submitted to placebo were analyzed for SIRT-1, H3K56ac, and p53ac content at baseline ( $\mathrm{t} 0)$ and at the trial end $(6 \mathrm{~m})$. Protein levels were normalized to beta actin, $\mathrm{H} 3$ and $\mathrm{p} 53$ content, respectively. The values reported represent the relative amount (ra) of protein expression obtained by densitometric analysis. b Cell extracts from T2DM patients' PBMCs (subject 7-12) submitted to resveratrol $40 \mathrm{mg} /$ day supplementation (Resv40) were lysed and analyzed for SIRT-1, $\mathrm{H} 3 \mathrm{~K} 56 \mathrm{ac}$, and p53ac content at baseline ( $\mathrm{t} 0)$ and at the trial end (6 m). Protein levels were normalized to beta actin, H3 and p53 content, respectively. The values reported represent the relative amount (ra) of protein expression obtained by densitometric analysis. c Cell extracts from T2DM patients' PBMCs (subject 13-18) submitted to resveratrol $500 \mathrm{mg} /$ day supplementation (Resv500) were lysed and analyzed for SIRT-1, $\mathrm{H} 3 \mathrm{~K} 56 \mathrm{ac}$, and p53ac content at baseline $(\mathrm{t} 0)$ and at the trial end $(6 \mathrm{~m})$. Protein levels were normalized to beta actin, $\mathrm{H} 3$, and $\mathrm{p} 53$ content, respectively. The values reported represent the relative amount (ra) of protein expression obtained by densitometric analysis

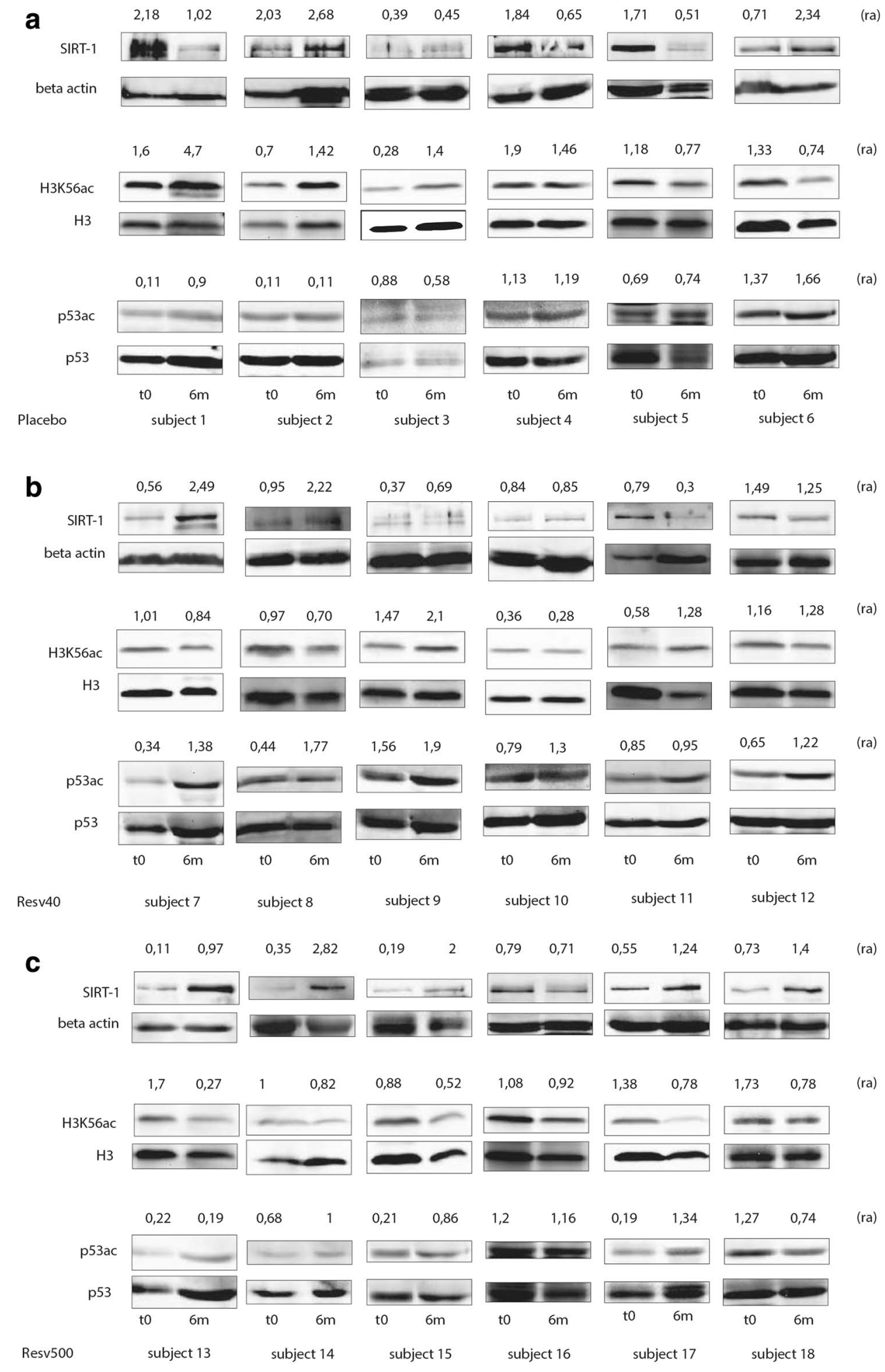

meaningful beneficial SIRT-1 effects on the inflammatory profile.

SIRT-1 controls the acetylation of transcriptional regulators involved in adipogenesis and fatty acid oxidation [31]. The anti-adipogenic effects of resveratrol have been demonstrated in preclinical models, while controversial results were found in humans $[12,13]$. We found a significant reduction in body fat percentage in individuals with higher SIRT-1 expression. However, this effect was lost when placebo patients were excluded. These results suggest 

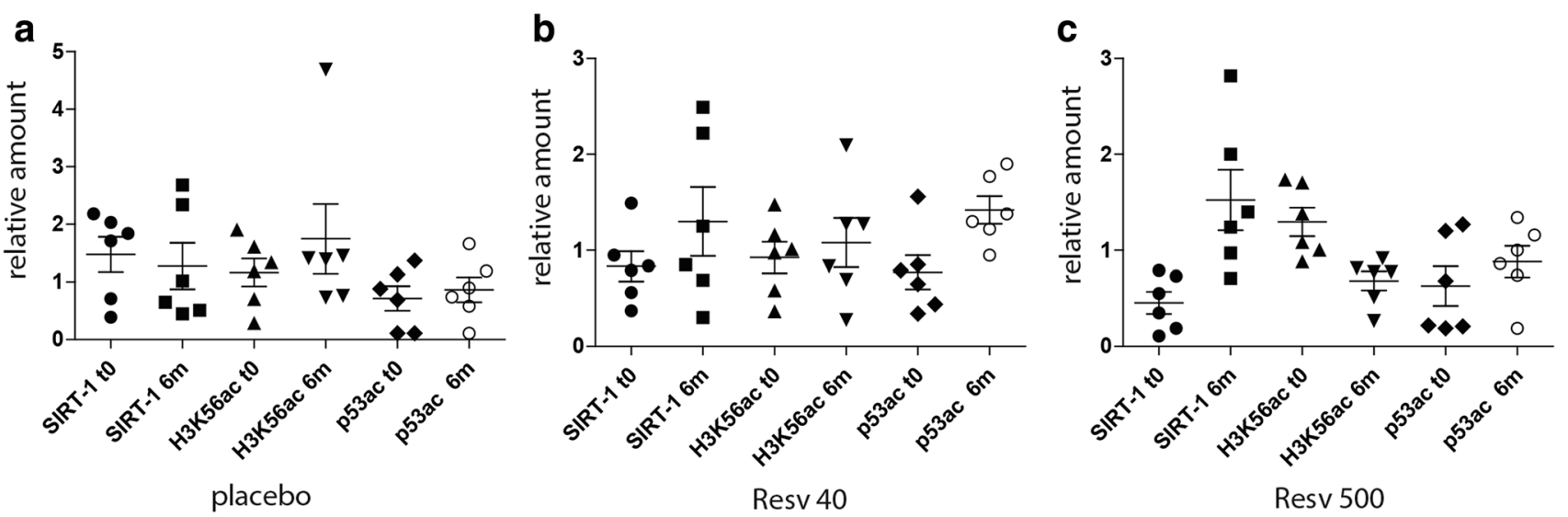

Fig. 2 Relative amount of SIRT-1, H3K56ac, and p53ac protein expression in PBMCs recovered from T2DM patients submitted to resveratrol supplementation or placebo. a The value reported represents the relative amount of protein expression obtained by densitometric analysis of cell extract analyzed in Fig. 1a. Cell extract from T2DM patient' PBMCs (subject 1-6) submitted to placebo was lysed and analyzed for SIRT-1, H3K56ac, and p53ac content at baseline $(\mathrm{t} 0)$ and at trial end $(6 \mathrm{~m})$. Protein levels were normalized to beta actin, H3, and p53 content, respectively. b The value reported represents the relative amount of protein expression obtained by densitometric analysis of cell extract analyzed in Fig. 1b. Cell extract from T2DM patient' PBMCs (subject 7-12) submitted to resveratrol $40 \mathrm{mg}$ /day supplementation (Resv40) was lysed and analyzed for SIRT-1, H3K56ac, and p53ac content at baseline (t0) and at trial end $(6 \mathrm{~m})$. Protein levels were normalized to beta actin, H3, and p53 content, respectively. $\mathbf{c}$ The value reported represents the relative amount of protein expression obtained by densitometric analysis of cell extract analyzed in Fig. 1c. Cell extract from T2DM patient' PBMCs (subject 13-18) submitted to resveratrol $500 \mathrm{mg} /$ day supplementation (Resv500) was lysed and analyzed for SIRT-1, H3K56ac, and p53ac content at baseline (t0) and at trial end $(6 \mathrm{~m})$. Protein levels were normalized to beta actin, $\mathrm{H} 3$, and $\mathrm{p} 53$ content, respectively
Table 4 Multiple logistic regression model. Associations between the highest tertile of delta SIRT-1 value and median value of delta $\mathrm{H} 3 \mathrm{~K} 56 \mathrm{ac}$ (upper), median value of TAS (middle), and delta percentage body fat (lower) in all arms of the trial (left) or including only individuals submitted to resveratrol supplementation (right)

\begin{tabular}{|c|c|c|c|c|c|c|}
\hline & \multicolumn{3}{|c|}{ All arms } & \multicolumn{3}{|c|}{ Resveratrol arms } \\
\hline & OR & $95 \% \mathrm{CI}$ & $P$ & OR & $95 \% \mathrm{CI}$ & $P$ \\
\hline Delta H3K56ac & 0.66 & $0.44-0.99$ & 0.04 & 0.67 & $0.43-1.04$ & 0.07 \\
\hline Age & 1.05 & $0.99-1.10$ & 0.10 & 1.02 & $0.96-1.08$ & 0.58 \\
\hline Male gender & 1.84 & $0.76-4.47$ & 0.17 & 1.27 & $0.49-3.31$ & 0.62 \\
\hline Treatment with resveratrol & 3.58 & $1.29-9.90$ & 0.01 & - & & \\
\hline Delta TAS & 1.01 & $1.00-1.02$ & 0.04 & 1.01 & $0.99-1.02$ & 0.05 \\
\hline Age & 1.04 & $0.99-1.10$ & 0.11 & 1.02 & $0.96-1.08$ & 0.50 \\
\hline Male gender & 2.02 & $0.83-4.95$ & 0.12 & 1.43 & $0.54-3.79$ & 0.47 \\
\hline Treatment with resveratrol & 3.09 & $1.16-8.22$ & 0.02 & - & & \\
\hline Delta percent body fat & 0.75 & $0.58-0.96$ & 0.02 & 0.81 & $0.63-1.05$ & 0.11 \\
\hline Age & 1.05 & $0.98-1.11$ & 0.14 & 1.02 & $0.95-1.08$ & 0.75 \\
\hline Male gender & 2.37 & $0.94-5.96$ & 0.07 & 1.58 & $0.59-4.21$ & 0.35 \\
\hline Treatment with resveratrol & 3.65 & $1.36-9.81$ & 0.01 & - & & \\
\hline
\end{tabular}

that SIRT-1 plays a role as an anti-adipogenic factor, while suggesting that different epigenetic mechanisms may be involved in SIRT-1-mediated anti-adipogenic effects.

SIRT-1 has also been shown to regulate glucose and lipid metabolism and therefore reduce cardiovascular risk factors $[30,31]$. We failed to find any association between SIRT-1 expression and metabolic variables. Highest SIRT-1 tertile patients showed increased $\mathrm{HbA}$ lc values at baseline and, a worse, though not significantly different metabolic profile overall. Compensatory SIRT-1 activation might be hypothesized; otherwise, it may be considered a casual finding.

\section{Clinical implications}

Although the physio-pathological mechanisms implicated are quite complex and multiple confounders may have biased our results, we herein provide evidence that boosting SIRT-1 expression translates into a significant reduction in $\mathrm{H} 3 \mathrm{~K} 56 \mathrm{ac}$, an epigenetic marker of oxidative stress-mediated damage, and into improvements in antioxidant activity. Understanding SIRT-1's role in the in vivo regulation of human metabolism and health still remains a challenging question and is worthy of further ad hoc clinical trials. Generally, advances in therapy have 
led to a significant decline in diabetes-associated complications; however, cardiovascular risk is still a relevant clinical problem. Recently, attention has been devoted to defining a diabetes epigenetic signature which would allow advance in developing new therapeutic options. The results of the present study suggest that histone deacetylase-based therapy could remove one epigenetic tag and change the ROS balance in diabetic patients. Thus, future efforts should be made to clearly assess and correlate the efficacy of epigenetic targeting approaches to the clinical outcomes in T2DM patients.

\section{Limitations}

Study power was originally calculated to detect an effect size of 0.5 on CRP value [17]. The sample size may have therefore been too small to find differences in subgroups. This was probably the case in analyses performed after excluding Placebo arm patients.

We did not measure plasmatic concentrations of resveratrol or its metabolites, meaning that actual substance exposure cannot be determined. Patient compliance could, however, be considered adequate, on the basis of phone calls, capsule counts and the consistent proportional increment in TAS levels with increasing resveratrol doses. The strengths of the present study were the large number of metabolic variables analyzed and the centralized, blindly analyzed, measurements.

\section{Conclusion}

These data suggest that SIRT-1-mediated changes in the epigenome and in the antioxidant response might impact on diabetes-associated risk factors.

Funding This study was supported by the Ministry of Health (Ricerca Sanitaria Finalizzata 2010; Grant Number RF-2010-2313155) and by the Ministry of Education, University and Research (PRIN 20102011 (Grant Number 2010JCWWKM_006) of Italy. Sponsors had no involvement in the study design, the collection, analysis and interpretation of data, in the writing of the report, and in the decision to submit the article for publication.

\section{Compliance with ethical standards}

Conflicts of interest The authors report no conflict of interest.

Ethical approval All procedures performed in studies involving human participants were in accordance with the ethical standards of the institutional and/or national research committee and with the 1964 Helsinki Declaration and its later amendments or comparable ethical standards.

Informed consent Informed consent was obtained from all individual participants included in the study.
Open Access This article is distributed under the terms of the Creative Commons Attribution 4.0 International License (http://creativecomm ons.org/licenses/by/4.0/), which permits unrestricted use, distribution, and reproduction in any medium, provided you give appropriate credit to the original author(s) and the source, provide a link to the Creative Commons license, and indicate if changes were made.

\section{References}

1. Meagher RB, Müssar KJ (2012) The influence of DNA sequence on epigenome-induced pathologies. Epigenetics Chromatin 5:11

2. Wegner M, Neddermann D, Piorunska-Stolzmann M, Jagodzinski PP (2014) Role of epigenetic mechanisms in the development of chronic complications of diabetes. Diabetes Res Clin Pract 105:164-175

3. Das C, Lucia MS, Hansen KC, Tyler JK (2009) CBP/p300-mediated acetylation of histone $\mathrm{H} 3$ on lysine 56 . Nature 459:113-117

4. Tjeertes JV, Miller KM, Jackson SP (2009) Screen for DNAdamage-responsive histone modifications identifies H3K9Ac and H3K56Ac in human cells. EMBO J 28:1878-1889

5. Lo KA, Bauchmann MK, Baumann AP et al (2011) Genomewide profiling of $\mathrm{H} 3 \mathrm{~K} 56$ acetylation and transcription factor binding sites in human adipocytes. PLoS ONE 6:e19778

6. Milne JC, Lambert PD, Schenk S et al (2007) Small molecule activators of SIRT1 as therapeutics for the treatment of type 2 diabetes. Nature 450:712-716

7. de Kreutzenberg SV, Ceolotto G, Papparella I et al (2010) Downregulation of the longevity-associated protein sirtuin 1 in insulin resistance and metabolic syndrome: potential biochemical mechanisms. Diabetes 59:1006-1015

8. Paneni F, Volpe M, Lüscher TF, Cosentino F (2013) SIRT1, p66(Shc), and Set7/9 in vascular hyperglycemic memory: bringing all the strands together. Diabetes 62:1800-1807

9. Togliatto G, Trombetta A, Dentelli P et al (2015) Unacylated ghrelin induces oxidative stress resistance in a glucose intolerance and peripheral artery disease mouse model by restoring endothelial cell miR-126 expression. Diabetes 64:1370-1382

10. Xia N, Daiber A, Förstermann U, Li H (2017) Antioxidant effects of resveratrol in the cardiovascular system. Br J Pharmacol 174:1633-1646

11. Tomé-Carneiro J, Larrosa M, González-Sarrías A, TomásBarberán FA, García-Conesa MA, Espín JC (2013) Resveratrol and clinical trials: the crossroad from in vitro studies to human evidence. Curr Pharmac Des 19:6064-6093

12. Ponzo V, Soldati L, Bo S (2014) Resveratrol: a supplementation for men or for mice? J Transl Med 12:158

13. Sahebkar A, Serban C, Ursoniu S et al (2015) Lack of efficacy of resveratrol on $\mathrm{C}$-reactive protein and selected cardiovascular risk factors - results from a systematic review and meta-analysis of randomized controlled trials. Int J Cardiol 189:47-55

14. Sahebkar A, Serban MC, Gluba-Brzózka A et al (2016) Lipid-modifying effects of nutraceuticals: an evidence-based approach. Nutrition 32:1179-1192

15. Cicero AFG, Colletti A, Bajraktari G et al (2017) Lipid lowering nutraceuticals in clinical practice: position paper from an International Lipid Expert Panel. Arch Med Sci 5:965-1005

16. Bo S, Ponzo V, Ciccone G et al (2016) Six months of resveratrol supplementation has no measurable effect in type 2 diabetic patients. A randomized, double blind, placebo-controlled trial. Pharmacol Res 111:896-905

17. Bo S, Ponzo V, Evangelista A et al (2017) Effects of 6 months of resveratrol $v s$ placebo on pentraxin 3 in patients with type 2 diabetes mellitus. A double-blind randomized-controlled trial. Acta Diabetol 54:499-507 
18. Trombetta A, Togliatto G, Rosso A et al (2013) Increase of palmitic acid concentration impairs endothelial progenitor cell and bone marrow-derived progenitor cell bioavailability: role of the STAT5/PPAR $\gamma$ transcriptional complex. Diabetes 62:1245-1257

19. Haigis MC, Guarente LP (2006) Mammalian sirtuins-emerging roles in physiology, aging, and calorie restriction. Genes Dev 20:2913-2921

20. Sosnowska B, Mazidi M, Penson P, Gluba-Brzózka A, Rysz J, Banach M (2017) The sirtuin family members SIRT-1, SIRT3 and SIRT6: their role in vascular biology and atherogenesis. Atherosclerosis 265:275-282

21. Vaziri H, Dessain SK, Ng Eaton E et al (2001) hSIR2(SIRT-1) functions as an NAD-dependent p53 deacetylase. Cell 107:149-159

22. Togliatto G, Dentelli P, Brizzi MF (2015) Skewed epigenetics: an alternative therapeutic option for diabetes complications. J Diabetes Res 2015:373708

23. Song SB, Jang SY, Kang HT et al (2017) Modulation of mitochondrial membrane potential and ROS generation by nicotinamide in a manner independent of SIRT1 and mitophagy. Mol Cells 40:503-514

24. Rayalam S, Della Fera MA, Baile CA (2011) Synergism between resveratrol and other phytochemicals: implication for obesity and osteoporosis. Mol Nutr Food Res 55:1177-1185
25. Côté CD, Rasmussen BA, Duca FA et al (2015) Resveratrol activates duodenal SIRT-1 to reverse insulin resistance in rats through a neuronal network. Nat Med 21:498-507

26. Cao D, Wang M, Qiu X et al (2015) Structural basis for allosteric, substrate-dependent stimulation of SIRT-1 activity by resveratrol. Gene Dev 29:1316-1325

27. González-Rodríguez Á, Santamaría B, Mas-Gutierrez JA et al (2015) Resveratrol treatment restores peripheral insulin sensitivity in diabetic mice in a SIRT-1-indipendent manner. Mol Nutr Food Res 59:1431-1442

28. Sathishkumar C, Prabu P, Balakumar M et al (2016) Augmentation of histone deacetylase 3 (HDAC3) epigenetic signature at the interface of proinflammation and insulin resistance in patients with type 2 diabetes. Clin Epigenetics 8:125

29. Yu J, Wu Y, Yang P (2016) High glucose-induced oxidative stress represses sirtuin deacetylase expression and increases histone acetylation leading to neural tube defects. J Neurochem 137:371-383

30. Ye X, Li M, Hou T, Gao T, Zhu W, Yang Y (2017) Sirtuins in glucose and lipid metabolism. Oncotarget 8:1845-1859

31. Picard F, Kurtev M, Chung N et al (2004) SIRT-1 promotes fat mobilization in while adipose tissue by repressing PPAR- $\gamma$. Nature 429:771-776 